\title{
Necessary and Sufficient Conditions of Optimality for a Damped Hyperbolic Equation in One-Space Dimension
}

\author{
Ismail Kucuk ${ }^{1,2}$ and Kenan Yildirim ${ }^{1}$ \\ ${ }^{1}$ Department of Mathematical Engineering, Yildiz Technical University, Istanbul, Turkey \\ ${ }^{2}$ Department of Mathematics and Statistics, American University of Sharjah, Sharjah, United Arab Emirates
}

Correspondence should be addressed to Kenan Yildirim; kenany@yildiz.edu.tr

Received 30 October 2013; Accepted 23 April 2014; Published 4 May 2014

Academic Editor: Youyu Wang

Copyright (C) 2014 I. Kucuk and K. Yildirim. This is an open access article distributed under the Creative Commons Attribution License, which permits unrestricted use, distribution, and reproduction in any medium, provided the original work is properly cited.

\begin{abstract}
The present paper deals with the necessary optimality condition for a class of distributed parameter systems in which the system is modeled in one-space dimension by a hyperbolic partial differential equation subject to the damping and mixed constraints on state and controls. Pontryagin maximum principle is derived to be a necessary condition for the controls of such systems to be optimal. With the aid of some convexity assumptions on the constraint functions, it is obtained that the maximum principle is also a sufficient condition for the optimality.
\end{abstract}

\section{Introduction}

It is well known that many processes in science and engineering are modeled by partial differential equations. The problems concerning the control of vibrating systems are generally governed by hyperbolic partial differential equations which are obtained by using conservation laws as a description of the distributed parameter system. In order to control these systems, the derivation of necessary conditions in the form of a maximum principle has been studied since the 1960s.

The Maximum principle is introduced for the first time by Pontryagin and his students as a necessary condition for the optimality of a mechanical system which is defined by ordinary differential or difference-differential equations [1]. Pontryagin's maximum principle is given in the form of a Hamiltonian that is defined in terms of an adjoint variable and the control function. The first application of maximum principle was the maximization of the terminal velocity of a rocket. Following this initial work, it is shown that the maximum principle is a necessary condition, if the value of the Hamiltonian maximized over the controls is concave in the state variables and the sufficient condition with appropriate transversality conditions [2]. Necessary conditions of optimality for distributed parameter systems described by boundary-value problems for hyperbolic and parabolic equations are studied in [3] where the completeness assumption on the class of admissible controls is imposed. In [4], Necessary condition for optimality is derived in a form similar to Pontryagin's maximum principle without admissible control function set which is bounded or closed. These necessary conditions are expressed in terms of certain "generalized Jocabians." For a general control problem formulated in terms of a differential inclusion, maximum principle is given by weak pseudo-Lipschitz behavior that is postulated on the underlying multifunction [5]. Necessary condition in the form of Pontryagin maximum principle by adopting the Dubovitskiy-Milyutin functional analytical approach is derived in [6]. In [7], Barnes presents a maximum principle as a necessary condition for optimal control of vibrating system that is modeled by second order linear hyperbolic PDE where completeness assumption is dropped and a regular point is used. Furthermore, Barnes shows that, by certain convexity assumptions on the constraint functions, the maximum principle is also a sufficient condition for the optimality of distributed parameters systems. Other related theoretical studies about the maximum principle in the literature are available such as [8-25]. In [1-7], the optimal necessary and sufficient conditions are derived for a single partial differential equation without damping term subject to the homogeneous boundary conditions in one space dimension. 
The systems that involve only one control function are studied in [1-25], while studies in [26-30] examine the systems with multiple control functions due to the size of the structures or to increase the control efficiency.

In the present paper, inspired by [7], the necessary optimality condition is given for a hyperbolic partial differential equation in one-space dimensional system. The system involves a damping and several control functions and is subject to the mixed integral constraints on state and control functions. The partial differential equation under consideration involves spatial derivatives of at most order four. The main goal of the control problem interested here is to minimize the performance index of the control problem in a given period of time with the control functions and state variable subject to the constraints in the form of integral equality or/and inequality. Under proper convexity assumptions on the constraint functions, the maximum principle is also a sufficient condition for a general class of hyperbolic partial differential equations in one-space dimensional system.

\section{Mathematical Formulation of the Problem}

Let us consider the following partial differential equation [31]:

$$
\begin{array}{r}
\mu(x) w_{t t}+N(x)[w]+M(x)\left[w_{t}\right]=E(x, t)+\sum_{j=1}^{N} f_{j}(x, t) \\
\text { on } \Omega=(0, \ell) \times\left(0, t_{f}\right),
\end{array}
$$

where $w(x, t)$ is the transversal displacement at $(x, t) \in \bar{\Omega}=$ $\left\{(x, t): x \in[0, \ell], t \in\left[0, t_{f}\right]\right\}, x$ is the space variable, $t$ is the time variable, $E(x, t)$ is the external excitation, $\mu(x)>0$ is the mass per unit length of the beam, $t_{f}$ is predetermined terminal time, and $f_{j}(x, t), j=1, \ldots, N$ are the control functions,

$$
\begin{aligned}
& M(x)[w]=\sum_{j=0}^{4} m_{j}(x)\left(\frac{\partial^{j} w}{\partial x^{j}}\right), \\
& N(x)[w]=\sum_{j=0}^{4} n_{j}(x)\left(\frac{\partial^{j} w}{\partial x^{j}}\right),
\end{aligned}
$$

in which $m_{j}(x)$ and $n_{j}(x)$ are continuous functions on $\Omega$, $M(x)$ and $N(x)$ are positive-definite operators, and $M(x)$ is the damping operator. Equation (1) is subject to the following boundary conditions:

$$
\begin{aligned}
& \left.a_{j}^{k}(x, t) \frac{\partial^{j} w}{\partial x^{j}}\right|_{x=0}=T_{j+k}(t), \quad k=1,2, j=0,1,2,3 \\
& \left.a_{j}^{k}(x, t) \frac{\partial^{j} w}{\partial x^{j}}\right|_{x=\ell}=S_{j+k}(t), \quad k=3,4, j=0,1,2,3,
\end{aligned}
$$

where $a_{j}^{k}(x, t), S_{j+k}(t)$, and $T_{j+k}(t)$ are continuous functions on $\Omega$ and the initial conditions

$$
\begin{aligned}
& w(x, 0)=w_{0}(x) \in H^{1}(0, \ell), \\
& w_{t}(x, 0)=w_{1}(x) \in L^{2}(0, \ell)
\end{aligned}
$$

in which

$$
H^{1}(0, \ell)=\left\{w_{0}(x) \in L^{2}(0, \ell): \frac{\partial w_{0}(x)}{\partial x} \in L^{2}(0, \ell)\right\} .
$$

The following assumptions are made:

$$
\begin{aligned}
& \text { (A1) } \partial^{j} w / \partial x^{j}, \partial^{j+1} w / \partial x^{j} \partial t, \partial^{2} w / \partial t^{2} \in L^{2}(\bar{\Omega}), j=0,1 \\
& \ldots, 4, \bar{\Omega} \text { is the closure of } \Omega ; \\
& \text { (A2) } a_{j}^{k}(x, t), T_{j+k}(t), S_{j+k}(t) \in L^{2}(\Omega) k=1, \ldots, 4, j= \\
& 0, \ldots, 3 ;
\end{aligned}
$$

(A3) the set of admissible control functions is given by

$$
\begin{aligned}
U_{\mathrm{ad}}=\left\{f_{k}(x, t) \mid f_{k}(x, t) \in L^{2}(\Omega),\right. & \\
& \left.\left|f_{k}(x, t)\right| \leq M_{k}<\infty, k=1, \ldots, N\right\} .
\end{aligned}
$$

in which $L^{2}(\Omega)$ denotes the Hilbert space of realvalued square-integrable functions on the domain $\Omega$ in the Lebesgue sense with the usual inner product and norm defined by

$$
\begin{gathered}
\langle f, g\rangle_{\Omega}=\int_{\Omega} f(x, t) g(x, t) d \Omega, \\
\|f\|^{2}=\langle f, f\rangle,
\end{gathered}
$$

respectively. Under these assumptions, the system equations (1)-(4) have a solution [32].

\section{Formulation of the Control Problem}

The optimal control problem aims to determine optimal control functions $f_{i}^{\circ}(x, t) \in U_{\text {ad }}, i=1, \ldots, N$ that minimizes the performance index at the terminal time $t_{f}$ :

$$
\begin{aligned}
\mathscr{J}_{0}( & \left.f_{1}(x, t), f_{2}(x, t), \ldots, f_{N}(x, t)\right) \\
= & \int_{0}^{\ell}\left[\mathscr{G}_{1}\left(x, w\left(x, t_{f}\right)\right)+\mathscr{G}_{2}\left(x, w_{t}\left(x, t_{f}\right)\right)\right] d x \\
& +\int_{0}^{t_{f}} \int_{0}^{\ell} \mathscr{G}_{0}\left(x, t, w(x, t), f_{1}(x, t), \ldots, f_{N}(x, t)\right) d x d t .
\end{aligned}
$$

The first two terms in the right hand side of (8) denote the modified energy of the system and the last term represents the control effort spent in control duration. $k$ th admissible 
control function $f_{k}^{\circ}(x, t)$ subject to (1)-(4) and the following constraints:

$$
\begin{aligned}
& \int_{0}^{\ell} h_{2 k}\left(x, w_{t}\left(x, t_{f}\right)\right) d x \\
& \quad+\int_{0}^{t_{f}} \int_{0}^{\ell} \mathscr{G}_{-2 k}\left(x, t, w(x, t), f_{k}(x, t)\right) d x d t=c_{-2 k} \\
& \int_{0}^{\ell} h_{1 k}\left(x, w\left(x, t_{f}\right)\right) d x \\
& \quad+\int_{0}^{t_{f}} \int_{0}^{\ell} \mathscr{G}_{-1 k}\left(x, t, w(x, t), f_{k}(x, t)\right) d x d t=c_{-1 k} \\
& \int_{0}^{t_{f}} \int_{0}^{\ell} \mathscr{G}_{i k}\left(x, t, w(x, t), f_{k}(x, t)\right) d x d t \leq c_{i k}, \quad 1 \leq i \leq m \\
& \int_{0}^{t_{f}} \int_{0}^{\ell} \mathscr{G}_{i k}\left(x, t, w(x, t), f_{k}(x, t)\right) d x d t=c_{i k}, \quad m<i \leq M,
\end{aligned}
$$

in which $h_{1 k}, h_{2 k}, \mathscr{G}_{0}, \mathscr{G}_{1}, \mathscr{G}_{2}$, and $\mathscr{G}_{i k}$, for $k=1,2, \ldots, N$, $i=-2,-1,1, \ldots, M$, are continuous functions of their parameters. Also, $h_{1 k}, \mathscr{G}_{0}, \mathscr{G}_{1}$, and $\mathscr{G}_{i k}$, for $k=1,2, \ldots, N, i=$ $-2,-1,1, \ldots, M$, have the continuous derivatives with respect to $w$ and $h_{2 k}, \mathscr{G}_{2}$ have the continuous derivatives with respect to $w_{t}$.

\section{Necessary and Sufficient Conditions for Optimality}

In this section, necessary condition of optimality is derived in the form of the maximum principle. Also, under proper convexity assumptions on the constraint functions, it is shown that maximum principle is also sufficient condition of optimality. For convenience, let us assume that

$$
\begin{array}{r}
\mathscr{G}_{0}\left(x, t, w, f_{1}(x, t), \ldots, f_{N}(x, t)\right) \\
=\sum_{k=1}^{N} \mathscr{G}_{0 k}\left(x, t, w, f_{k}(x, t)\right),
\end{array}
$$

where $\mathscr{G}_{0 k}\left(x, t, w, f_{k}(x, t)\right)$ is the related term with $k$ th control function in $\mathscr{G}_{0}$. In order to achieve the maximum principle, an adjoint variable $v(x, t)$ along with the adjoint operator is introduced. The adjoint variable $v(x, t)$ satisfies the following equation:

$$
\begin{aligned}
\mu(x) & v_{t t}+N^{*}(x)[v]-M^{*}(x)\left[v_{t}\right] \\
= & \sum_{k=1}^{N} \sum_{i=-2}^{M} \lambda_{i k} \frac{\partial \mathscr{G}_{i k}}{\partial w}\left(x, t, w, f_{k}(x, t)\right), \quad \lambda_{0 k}=\lambda_{0},
\end{aligned}
$$

where we introduced the Lagrange multiplier $\lambda_{i k} \leq 0$ and

$$
\begin{aligned}
& M^{*}(x)[v]=\sum_{j=0}^{4}(-1)^{j}\left(\frac{\partial^{j}}{\partial x^{j}}\right)\left(v m_{j}(x)\right), \\
& N^{*}(x)[v]=\sum_{j=0}^{4}(-1)^{j}\left(\frac{\partial^{j}}{\partial x^{j}}\right)\left(v n_{j}(x)\right) .
\end{aligned}
$$

Equation (11) is subject to the following homogeneous boundary conditions:

$$
\begin{aligned}
& \left.a_{j}^{k}(x, t) \frac{\partial^{j} v}{\partial x^{j}}\right|_{x=0}=0, \quad k=1,2, j=0,1,2,3 \\
& \left.a_{j}^{k}(x, t) \frac{\partial^{j} v}{\partial x^{j}}\right|_{x=\ell}=0, \quad k=3,4, j=0,1,2,3
\end{aligned}
$$

and the terminal conditions

$$
\begin{aligned}
& v\left(x, t_{f}\right)=\frac{1}{\mu(x)} \lambda_{0} \frac{\partial \mathscr{G}_{2}}{\partial w_{t}}\left(x, w_{t}\left(x, t_{f}\right)\right) \\
& +\frac{1}{\mu(x)} \sum_{k=1}^{N} \lambda_{-2 k} \frac{\partial h_{2 k}}{\partial w_{t}}\left(x, w_{t}\left(x, t_{f}\right)\right), \\
& v_{t}\left(x, t_{f}\right)-M^{*}(x)[v]\left(x, t_{f}\right) \\
& =-\frac{1}{\mu(x)} \sum_{k=1}^{N} \lambda_{-1 k} \frac{\partial h_{1 k}}{\partial w}\left(x, w\left(x, t_{f}\right)\right) \\
& -\frac{1}{\mu(x)} \lambda_{0} \frac{\partial \mathscr{G}_{1}}{\partial w}\left(x, w\left(x, t_{f}\right)\right) .
\end{aligned}
$$

Let us introduce a special perturbation of the optimal control problem and three lemmas to derive the maximum principle. Suppose that $f_{k}^{\circ}(x, t), k=1, \ldots, N$ are optimal control functions corresponding to optimal displacement $w^{\circ}$. Let $\left(x_{1}, t_{1}\right), \ldots,\left(x_{P}, t_{P}\right)$ be $P$ arbitrary points in the open region $\Omega$ and let $f_{k j}(x, t), k=1, \ldots, N, j=1, \ldots, P$ be $P$ arbitrary subfunctions for every admissible control function $f_{k} \in U_{\mathrm{ad}}, k=1,2, \ldots, N$. Also, let us assume that $x_{1} \leq$ $x_{2} \leq \cdots \leq x_{P}$. Choose $\varsigma>0$ such that $x_{i}+P \varsigma<x_{j}$, if $x_{i}<x_{j}, x_{P}+P \varsigma<\ell$ and $t_{i}+\varsigma<t_{f}$, for each $0 \leq i \leq P$. Let $\varepsilon_{1}, \ldots, \varepsilon_{P}$ be real parameters satisfying $0 \leq \varepsilon_{j} \leq \varsigma^{2}$. Let $X_{1}=x_{1}$ and $X_{j}=x_{j}+\sqrt{\varepsilon_{1}}+\cdots+\sqrt{\varepsilon_{j-1}}$ be for $1<j \leq P$. Hence, the intervals $X_{j} \leq x \leq X_{j}+\sqrt{\varepsilon_{j}}$ and the rectangles $R_{j}:\left[X_{j}, X_{j}+\sqrt{\varepsilon_{j}}\right] \times\left[t_{j}, t_{j}+\sqrt{\varepsilon_{j}}\right]$ do not have any intersection for $1 \leq j \leq P$, respectively. $\varepsilon$ denotes the vector $\left(\varepsilon_{1}, \ldots, \varepsilon_{P}\right) \in \mathbb{R}^{P} ; \mathbb{R}^{P}$ is $P$-dimensional Euclidean space, and the norm of $\varepsilon$ is given by $|\varepsilon|=\varepsilon_{1}+\cdots+\varepsilon_{P}$. The admissible controls $f_{k \varepsilon}(x, t) \in \bar{\Omega}$ are defined by

$$
f_{k \varepsilon}(x, t)= \begin{cases}f_{k}^{\circ}(x, t), & \text { if }(x, t) \notin \bigcup_{j=1}^{P} R_{j}, \\ f_{k j}(x, t), & \text { if }(x, t) \in R_{j}, j=1, \ldots, P,\end{cases}
$$

for $k=1,2, \ldots, N$

Lemma 1. Let $w_{\varepsilon}$ satisfy the system given by (1)-(4) corresponding to the controls $f_{1 \varepsilon}(x, t), f_{2 \varepsilon}(x, t), \ldots, f_{N \varepsilon}(x, t)$. Consider the following difference functions,

$$
\begin{aligned}
& \Delta F(x, t)=F_{\varepsilon}(x, t)-F^{\circ}(x, t), \\
& \Delta w(x, t)=w_{\varepsilon}(x, t)-w^{\circ}(x, t),
\end{aligned}
$$


in which

$$
F(x, t)=\sum_{k=1}^{N} f_{k}(x, t) .
$$

Note that $\Delta w(x, t)$ satisfies following equation:

$$
\mu(x) \Delta w_{t t}+N(x)[\Delta w]+M(x)\left[\Delta w_{t}\right]=\Delta F(x, t),
$$

and the following homogeneous boundary conditions:

$$
\begin{aligned}
& \left.b_{j}^{k}(x, t) \frac{\partial^{j} \Delta w}{\partial x^{j}}\right|_{x=0}=0, \quad k=1,2, j=0,1,2,3 \\
& \left.b_{j}^{k}(x, t) \frac{\partial^{j} \Delta w}{\partial x^{j}}\right|_{x=\ell}=0, \quad k=3,4, j=0,1,2,3
\end{aligned}
$$

and also, the zero initial conditions:

$$
\Delta w(x, 0)=0, \quad \Delta w_{t}(x, 0)=0 .
$$

Then,

$$
\begin{gathered}
\int_{0}^{\ell} \Delta w^{2}\left(x, t_{f}\right) d x=o(\varepsilon), \quad \int_{0}^{\ell} \Delta w_{t}^{2}\left(x, t_{f}\right) d x=o(\varepsilon), \\
\int_{0}^{t_{f}} \int_{0}^{\ell} \Delta w^{2}(x, t) d x d t=o(\varepsilon) .
\end{gathered}
$$

$o(\varepsilon)$ is a quantity such that

$$
\lim _{\varepsilon \rightarrow 0}\left(\frac{o(\varepsilon)}{|\varepsilon|}\right)=0 .
$$

Proof. We start the proof by examining the following energy integral:

$$
E(t)=\frac{1}{2} \int_{0}^{\ell}\left\{\left(\mu(x) \Delta w_{t}\right)^{2}+\frac{1}{2} N(x)[\Delta w]^{2}\right\} d x
$$

then, it can be rewritten in [33] as

$$
\begin{aligned}
E(t)= & \int_{0}^{t} \frac{d E(\tau)}{d \tau} d \tau \\
= & \int_{0}^{t} \frac{1}{2} \int_{0}^{\ell}\left\{2 \mu(x) \Delta w_{t} \Delta w_{t t}\right. \\
& \left.\quad+\frac{1}{2} N(x)\left[2 \Delta w \Delta w_{t}\right]\right\} d x d \tau .
\end{aligned}
$$

With integration by parts and using homogeneous boundary conditions given by (19a) and (19b), (24) becomes

$$
\begin{aligned}
E(t) & =\int_{0}^{t} \int_{0}^{\ell}\left\{\mu(x) \Delta w_{t t}+N(x)[\Delta w]\right\} \Delta w_{t} d x d \tau \\
& =\int_{0}^{t} \int_{0}^{\ell}\left\{\Delta F(x, \tau) \Delta w_{t}-\Delta w_{t} M(x)\left[\Delta w_{t}\right]\right\} d x d \tau \\
& \leq \int_{0}^{t} \int_{0}^{\ell} \Delta F(x, \tau) \Delta w_{t}(x, \tau) d x d \tau .
\end{aligned}
$$

Note that if $N(x)$ is nonself adjoint operator, the foregoing inequality is also satisfied. By applying the Cauchy-Schwartz inequality to the space integral, we obtain

$$
\begin{aligned}
E(t) & \leq \int_{0}^{t}\left[\int_{0}^{\ell}\left(\Delta w_{t}\right)^{2} d x\right]^{1 / 2}\left[\int_{0}^{\ell}(\Delta F(x, \tau))^{2} d x\right]^{1 / 2} d \tau \\
& \leq \int_{0}^{t} E^{1 / 2}(\tau)\left[\int_{0}^{\ell}(\Delta F(x, \tau))^{2} d x\right]^{1 / 2} d \tau .
\end{aligned}
$$

Taking the sup of both sides of (26) leads to

$$
\begin{aligned}
\sup E(t) & \leq \sup E^{1 / 2}(t) \int_{0}^{t}\left[\int_{0}^{\ell}(\Delta F(x, \tau))^{2} d x\right]^{1 / 2} d \tau \\
& =\sup E^{\frac{1}{2}}(t) \sum_{i=1}^{P} o\left(\varepsilon_{i}^{5 / 4}\right)
\end{aligned}
$$

where $o(r)$ is a quantity such that

$$
\lim _{r \rightarrow 0^{+}}\left(\frac{o(r)}{r}\right)=\text { constant. }
$$

By means of (27), the following inequality is observed for each $t \in\left[0, t_{f}\right]$ :

$$
0 \leq E^{1 / 2}(t) \leq o\left(\varepsilon^{5 / 4}\right) .
$$

Because 5/4 > 0 [34], the following equality is obtained:

$$
E(t)=o(\varepsilon) .
$$

Because the coefficients of (1) are bounded away from zero, the conclusion of Lemma 1 is obtained from (30). It is concluded from Lemma 1 that

$$
\lim _{\Delta F(x, t) \rightarrow 0} \Delta w(x, t)=0 .
$$

Namely, (1)-(4) have a unique solution.

Let us define the differential operator $\mathscr{L}$ and its adjoint operator $\mathscr{M}$ as follows:

$$
\begin{aligned}
& \mathscr{L}[w] \equiv \mu(x) w_{t t}+N(x)[w]+M(x)\left[w_{t}\right], \\
& \mathscr{M}[v] \equiv \mu(x) v_{t t}+N^{*}(x)[v]-M^{*}(x)\left[v_{t}\right] .
\end{aligned}
$$

Lemma 2. Let $v$ and $\Delta w(x, t)=w(x, t)-w^{\circ}(x, t)$ be two functions which are defined in $L^{2}(\Omega)$. Also, let us assume that $v$ and $\Delta w(x, t)$ satisfy conditions (13a) and (13b) and (19a) and (19b)-(20), respectively. Then,

$$
\begin{aligned}
\int_{0}^{t_{f}} \int_{0}^{\ell}\{v \mathscr{L}[\Delta w]-\Delta w \mathscr{M}[v]\} d x d t \\
=\int_{0}^{\ell} \mu(x)\left\{v\left(x, t_{f}\right) \Delta w_{t}\left(x, t_{f}\right)-\Delta w\left(x, t_{f}\right)\right. \\
\left.\quad \times\left\{v_{t}\left(x, t_{f}\right)-M^{*}(x)\left[v\left(x, t_{f}\right)\right]\right\}\right\} d x
\end{aligned}
$$


Proof. The reader is referred to [31].

Definition 3. For the arbitrary constants, $\lambda_{-2 k}, \lambda_{-1 k}, \lambda_{0}, \ldots$, $\lambda_{M k}, k=1, \ldots, N, v$ is the solution of the system equations (11)-(14a) and (14b). Let $w^{\circ}$ be the response function corresponding to optimal control functions $f_{k}^{\circ} \in U_{\text {ad }}, k=1$, $\ldots, N, u$ be any of the following functions:

$$
\begin{gathered}
u(x, t)=\mathscr{G}_{i k}\left(x, t, w, f_{k}(x, t)\right), \quad f_{k} \in U_{\mathrm{ad}} \text { is fixed } \\
u(x, t)=\mathscr{G}_{i k}\left(x, t, w^{\circ}, f_{k}^{\circ}(x, t)\right), \\
u(x, t)=\sum_{k=1}^{N} v f_{k}^{\circ}, \quad f_{k}^{\circ} \in U_{\mathrm{ad}}, \\
u(x, t)=\sum_{k=1}^{N} v f_{k}, \quad f_{k} \in U_{\mathrm{ad}} \text { is fixed. }
\end{gathered}
$$

A point $(\bar{x}, \bar{t})$ is called a regular point, for each $f_{k}, k=$ $1, \ldots, N \in U_{\text {ad }}$, if it satisfies the following equality for any sufficiently small $\varepsilon>0$ :

$$
\int_{\bar{t}}^{\bar{t}+\sqrt{\varepsilon}} \int_{\bar{x}}^{\bar{x}+\sqrt{\varepsilon}} u(x, t) d x d t=\varepsilon u(\bar{x}, \bar{t})+o(\varepsilon) .
$$

It can be concluded from [35] that all points of $[0, \ell] \times\left[0, t_{f}\right]$ are regular for each $f_{k}, k=1, \ldots, N \in U_{\text {ad }}$.

Let $\mathscr{F}_{k}$ and $Z$ denote the vector valued functional $\left(\mathscr{F}_{-2 k}, \mathscr{J}_{-1 k}, \mathscr{J}_{0 k}, \ldots, \mathscr{J}_{M k}\right)$, for $k=1, \ldots, N$, and the set

$$
Z=\left\{\mathscr{f}_{k}\left(f_{k}\right): f_{k} \in U_{\mathrm{ad}}\right\} \subset \mathbb{R}^{M+3} .
$$

Definition 4. If there exists a surface in the following form:

$$
\mathscr{J}_{k \varepsilon}=\mathscr{J}_{k}\left(f_{k}^{\circ}\right)+\sum_{j=1}^{P} d_{k j} \varepsilon_{j}+o(\varepsilon)
$$

in $Z$ for sufficiently small $\varepsilon_{j}$ and $d_{k 1}, \ldots, d_{k P}$ are any finite collection of vectors from $D$, then the set $D$ is called as a derived set of the set $Z$ at $\mathscr{J}_{k}\left(f_{k}^{\circ}\right)$ [36].

Lemma 5. Assume that the points $\left(x_{i}, t_{i}\right)$ are regular points in $\Omega$, for $i=1,2, \ldots, P$, and $\overline{\mathscr{J}}_{k}\left(f_{k}\right)$ is introduced as follows, for any $f_{k} \in U_{a d}, k=1, \ldots, N$ :

$$
\begin{aligned}
\overline{\mathscr{J}}_{k}\left(f_{k}\right)=\int_{0}^{\ell}\{ & \left(\frac{\lambda_{0}}{N}\right) \\
& \times\left[\mathscr{G}_{1}\left(x, w\left(x, t_{f}\right)\right)+\mathscr{G}_{2}\left(x, w_{t}\left(x, t_{f}\right)\right)\right] \\
+ & {\left[\lambda_{-2 k} h_{2 k}\left(x, w_{t}\left(x, t_{f}\right)\right)\right.} \\
& \left.\left.+\lambda_{-1 k} h_{1 k}\left(x, w\left(x, t_{f}\right)\right)\right]\right\} d x \\
+ & \int_{0}^{t_{f}} \int_{0}^{\ell} \sum_{i=-2}^{M}\left[\lambda_{i k} \mathscr{G}_{i k}\left(x, t, w, f_{k}\right)\right] d x d t .
\end{aligned}
$$

If $P=1$, for $k=1, \ldots, N f_{k} \in U_{a d}$, there exist constants $\lambda_{-2 k}, \lambda_{-1 k}, \lambda_{0}, \ldots, \lambda_{M k}$ (not all zero) such that

$$
\begin{gathered}
\lambda_{0} \leq 0, \quad \lambda_{i k} \leq 0 \quad(0 \leq i \leq m), \\
\lim _{\varepsilon \rightarrow 0^{+}} \frac{\overline{\mathscr{J}}_{k}\left(f_{k \varepsilon}\right)-\overline{\mathscr{J}}_{k}\left(f_{k}^{\circ}\right)}{\varepsilon} \leq 0,
\end{gathered}
$$

in which $f_{k}^{\circ}$ 's and $f_{\varepsilon k}$ 's are functions which are defined in (15).

Proof. Define the functionals $\mathscr{J}_{-2 k}, \mathscr{J}_{-1 k}, \mathscr{F}_{1 k}, \ldots, \mathscr{F}_{M k}$ on the class of admissible controls by

$$
\begin{aligned}
& \mathscr{J}_{-2 k}\left(f_{k}\right)= \int_{0}^{\ell} h_{2 k}\left(x, w_{t}\left(x, t_{f}\right)\right) d x \\
&+\int_{0}^{t_{f}} \int_{0}^{\ell} G_{-2 k}\left(x, t, w, f_{k}\right) d x d t, \\
& \mathscr{J}_{-1 k}\left(f_{k}\right)= \int_{0}^{\ell} h_{1 k}\left(x, w_{t}\left(x, t_{f}\right)\right) d x \\
&+\int_{0}^{t_{f}} \int_{0}^{\ell} G_{-1 k}\left(x, t, w, f_{k}\right) d x d t, \\
& \mathscr{F}_{i k}\left(f_{k}\right)=\int_{0}^{t_{f}} \int_{0}^{\ell} G_{i k}\left(x, t, w, f_{k}\right) d x d t, \quad i=1, \ldots, M .
\end{aligned}
$$

In order to use the Lagrange multiplier rule, we should construct a derived set $D$ for the set $Z$ at $\mathscr{J}_{k}\left(f_{k}^{\circ}\right)$ [36]. To this end, functions $v_{j k}$ are introduced, for $k=1,2, \ldots, N, j=$ $-2,-1,1, \ldots, M$, satisfying

$$
\begin{gathered}
\mu(x) \frac{\partial^{2} v_{j k}}{\partial t^{2}}+N^{*}(x)\left[v_{j k}\right]-M^{*}(x)\left[\frac{\partial v_{j k}}{\partial t}\right] \\
=\frac{\partial \mathscr{G}_{j k}}{\partial w}\left(x, t, w, f_{k}\right), \quad 0 \leq x \leq \ell, 0 \leq t \leq t_{f}, \\
\left.a_{i}^{\alpha}(x, t) \frac{\partial^{i} v_{j k}}{\partial x^{i}}\right|_{x=0}=0, \\
\alpha=1,2, \quad i=0,1,2,3 \quad-2 \leq j \leq M, \quad k=1, \ldots, N, \\
\alpha=3,4, \quad i=0,1,2,3 \quad-2 \leq j \leq M, \quad k=1, \ldots, N, \\
\left.a_{i}^{\alpha}(x, t) \frac{\partial^{i} v_{j k}}{\partial x^{i}}\right|_{x=\ell}=0, \\
\begin{array}{c}
\frac{v_{-2 k}}{}\left(x, t_{f}\right)=\frac{1}{\mu(x)} \frac{\partial h_{2 k}}{\partial w_{t}}\left(x, w_{t}\left(x, t_{f}\right)\right), \\
\frac{\partial v_{-2 k}}{\partial t}\left(x, t_{f}\right)=0, \\
v_{-1 k}\left(x, t_{f}\right)=0, \\
\frac{\partial v_{-1 k}}{\partial t}\left(x, t_{f}\right)=\frac{-1}{\mu(x)} \frac{\partial h_{1 k}}{\partial w}\left(x, w\left(x, t_{f}\right)\right), \\
v_{j k}\left(x, t_{f}\right)=0, \quad \frac{\partial v_{j k}}{\partial t}\left(x, t_{f}\right)=0, \\
j=1, \ldots, M, \quad k=1, \ldots, N
\end{array}
\end{gathered}
$$


and, for $k=1, \ldots, N$ and $j=0$, satisfying

$$
\begin{aligned}
& \mu(x) \frac{\partial^{2} v_{0 k}}{\partial t^{2}}+N^{*}(x)\left[v_{0 k}\right]-M^{*}(x)\left[\frac{\partial v_{0 k}}{\partial t}\right] \\
&=\frac{1}{N} \frac{\partial \mathscr{G}_{0}}{\partial w}\left(x, t, w, f_{k}\right), \\
& v_{0 k}\left(x, t_{f}\right)=\frac{1}{N \mu(x)} \frac{\partial \mathscr{G}_{2}}{\partial w_{t}}\left(x, w_{t}\left(x, t_{f}\right)\right) \\
& \frac{\partial v_{0 k}}{\partial t}\left(x, t_{f}\right)-M^{*}(x)\left[v_{0 k}\right]\left(x, t_{f}\right) \\
&=\frac{-1}{N \mu(x)} \frac{\partial \mathscr{G}_{1}}{\partial w}\left(x, w\left(x, t_{f}\right)\right) .
\end{aligned}
$$

For each point, $(x, t) \in(0, \ell) \times\left(0, t_{f}\right), i=-2,-1,0, \ldots$, $M, k=1, \ldots, N, d_{k}^{i}\left(x, t, \bar{f}_{k}\right)$ is defined as follows:

$$
\begin{aligned}
d_{k}^{i}\left(x, t, \bar{f}_{k}\right)= & v_{i k}(x, t)\left(\bar{f}_{k}-f_{k}^{\circ}\right)+\mathscr{G}_{i k}\left(x, t, w^{\circ}(x, t), \bar{f}_{k}\right) \\
& -\mathscr{G}_{i k}\left(x, t, w^{\circ}(x, t), f_{k}^{\circ}\right) .
\end{aligned}
$$

To show that

$$
\begin{gathered}
D=\left\{d_{k} \mid d_{k}=\left(d_{k}^{-2}\left(x, t, \bar{f}_{k}\right), \ldots, d_{k}^{M}\left(x, t, \bar{f}_{k}\right)\right),\right. \\
\left.(x, t) \text { a regular point of } f_{k}^{\circ}, \bar{f}_{k} \in U_{\mathrm{ad}}\right\}
\end{gathered}
$$

is a derived set for $Z$ at $\mathscr{J}_{k}\left(f_{k}^{\circ}\right)$, let $d_{k 1}, d_{k 2}, \ldots, d_{k P}$ be an arbitrary finite collection of vectors from $D$. We must show that there exist points $\mathscr{J}_{\varepsilon k} \in Z$, which are subject to the vector parameter $\varepsilon=\left(\varepsilon_{1}, \ldots, \varepsilon_{P}\right)$ for all sufficiently small positive values of $\varepsilon$ such that

$$
\mathscr{J}_{\varepsilon k}=\mathscr{J}_{k}\left(f_{k}^{\circ}\right)+\sum_{j=1}^{P} d_{k j} \varepsilon_{j}+o(\varepsilon) .
$$

Since $d_{k j} \in D, j=1, \ldots, P k=1, \ldots, N$, there exist $\left(x_{1}, t_{1}\right), \ldots,\left(x_{P}, t_{P}\right)$ regularity points of $f_{k}^{\circ}$ and subfunctions $f_{k 1}, \ldots, f_{k P} \in U_{\text {ad }}$ such that

$$
\begin{array}{r}
d_{k j}=\left(d_{k}^{-2}\left(x_{j}, t_{j}, f_{k j}\right), \ldots, d_{k}^{M}\left(x_{j}, t_{j}, f_{k j}\right)\right), \\
j=1, \ldots, P, \quad k=1, \ldots, N .
\end{array}
$$

To show that $\mathscr{J}_{\varepsilon k}$ can be written as $\mathscr{J}_{\varepsilon k}=\mathscr{J}_{k}\left(f_{k \varepsilon}\right)$ where $f_{k \varepsilon}$ is the admissible control given by (15), for $i=1, \ldots, M$, we observe that

$$
\begin{aligned}
& \mathscr{F}_{i k}\left(f_{k \varepsilon}\right)-\mathscr{J}_{i k}\left(f_{k}^{\circ}\right) \\
& =\int_{0}^{t_{f}} \int_{0}^{\ell}\left[\mathscr{G}_{i k}\left(x, t, w_{\varepsilon}(x, t), f_{k \varepsilon}(x, t)\right)\right. \\
& \left.\quad-\mathscr{G}_{i k}\left(x, t, w^{\circ}(x, t), f_{k}^{\circ}(x, t)\right)\right] d x d t \\
& =\int_{0}^{t_{f}} \int_{0}^{\ell}\left[\mathscr{G}_{i k}\left(x, t, w_{\varepsilon}(x, t), f_{k \varepsilon}(x, t)\right)\right. \\
& +\int_{0}^{t_{f}} \int_{0}^{\ell}\left[\mathscr{G}_{i k}\left(x, t, w^{\circ}(x, t), f_{k \varepsilon}(x, t)\right)\right. \\
& \left.-\mathscr{G}_{i k}\left(x, t, w^{\circ}(x, t), f_{k}^{\circ}(x, t)\right)\right] d x d t
\end{aligned}
$$

$$
\begin{aligned}
& =\int_{0}^{t_{f}} \int_{0}^{\ell} \frac{\partial \mathscr{G}_{i k}}{\partial w}\left(x, t, w^{\circ}, f_{k}^{\circ}(x, t)\right) \Delta w(x, t) d x d t \\
& +\sum_{j=1}^{P} \varepsilon_{j}\left[\mathscr{G}_{i k}\left(x_{j}, t_{j}, w^{\circ}\left(x_{j}, t_{j}\right), f_{k j}\right)\right. \\
& \left.\quad-\mathscr{G}_{i k}\left(x_{j}, t_{j}, w^{\circ}\left(x_{j}, t_{j}\right), f_{k j}^{\circ}\left(x_{j}, t_{j}\right)\right)\right] \\
& +\sum_{j=1}^{P} o\left(\varepsilon_{j}\right) .
\end{aligned}
$$

In order to obtain (47), we use that $f_{k}^{\circ}$ is regular at each of the points $\left(x_{j}, t_{j}\right)$ and we use the conclusion of Lemma 1 . If the following equality is substituted in (33)

$$
\begin{aligned}
\mathscr{M} v_{k i} & =\frac{\partial \mathscr{G}_{i k}}{\partial w}\left(x, t, w^{\circ}(x, t), f_{k}^{\circ}\right), \\
i & =1, \ldots, M, \quad k=1, \ldots, N,
\end{aligned}
$$

it is observed that

$$
\begin{aligned}
& \int_{0}^{\ell} \int_{0}^{t_{f}} \Delta w(x, t) \mathscr{M} v_{k i} d x d t \\
& \quad=\int_{0}^{\ell} \int_{0}^{t_{f}} v_{k i}(x, t)\left(f_{k \varepsilon}(x, t)-f_{k}^{\circ}(x, t)\right) \\
& \quad=\sum_{j=1}^{P} \varepsilon_{j} v_{k i}\left(x_{j}, t_{j}\right)\left(f_{k j}-f_{k j}^{\circ}\left(x_{j}, t_{j}\right)\right)+o(\varepsilon) .
\end{aligned}
$$

By means of (43) and (47), we can write

$$
\begin{array}{r}
\mathscr{J}_{i k}\left(f_{k \varepsilon}\right)=\mathscr{J}_{i k}\left(f_{k}^{\circ}\right)+\sum_{j=1}^{P} d_{k j}^{i} \varepsilon_{j}+o(\varepsilon), \\
i=1, \ldots, M, \quad k=1, \ldots, N,
\end{array}
$$

where $d_{k j}^{i}$ denotes the $i$ th component of $d_{k j}$. For $i=0$, we have

$$
\begin{aligned}
\mathscr{J}_{0 k}\left(f_{k \varepsilon}\right)-\mathscr{J}_{0 k}\left(f_{k}^{\circ}\right) & \\
=\int_{0}^{\ell} & {\left[\frac{1}{N} \frac{\partial \mathscr{G}_{1}}{\partial w}\left(x, w^{\circ}\left(x, t_{f}\right)\right) \Delta w\left(x, t_{f}\right)\right.} \\
& \left.+\frac{1}{N} \frac{\partial \mathscr{G}_{2}}{\partial w_{t}}\left(x, w_{t}^{\circ}\left(x, t_{f}\right)\right) \Delta w_{t}\left(x, t_{f}\right)\right] d x \\
+ & \sum_{j=1}^{P} \varepsilon_{j}\left[\mathscr{G}_{0 k}\left(x_{j}, t_{j}, w_{\varepsilon}\left(x_{j}, t_{j}\right), f_{k j}\right)\right. \\
+ & \left.-\int_{0}^{\ell} \int_{0}^{t_{f}}\left(\mathscr{M}_{0 k}, t_{j}, w_{\varepsilon}\left(x_{j}, t_{j}\right), f_{k j}^{\circ}\right)\right]
\end{aligned}
$$


in which $\mathscr{J}_{0 k}=(1 / N) \int_{0}^{\ell}\left[\mathscr{G}_{1}\left(x, w\left(x, t_{f}\right)\right)+\mathscr{G}_{2}\left(x, w_{t}(x\right.\right.$, $\left.\left.\left.t_{f}\right)\right)\right] d x+\int_{0}^{t_{f}} \int_{0}^{\ell} \mathscr{G}_{0 k}\left(x, t, w, f_{k}\right) d x d t$. Considering (33) and (41a), (41b), (41c), (41d), (41e), and (41f), it is observed that

$$
\begin{aligned}
\int_{0}^{t_{f}} & \int_{0}^{\ell} \Delta w(x, t) \mathscr{M} v_{0 k} d x d t \\
= & \int_{0}^{t_{f}} \int_{0}^{\ell} v_{0 k}(x, t)\left(f_{k \varepsilon}(x, t)-f_{k}^{\circ}(x, t)\right) d x d t \\
& -\int_{0}^{\ell}\left[\frac{1}{N} \frac{\partial \mathscr{G}_{1}}{\partial w}\left(x, w^{\circ}\left(x, t_{f}\right)\right) \Delta w\left(x, t_{f}\right)\right. \\
& \left.+\frac{1}{N} \frac{\partial \mathscr{G}_{2}}{\partial w_{t}}\left(x, w_{t}\left(x, t_{f}\right)\right) \Delta w_{t}\left(x, t_{f}\right)\right] .
\end{aligned}
$$

If (52) is substituted into (51), (50) is obtained for $i=0, k=$ $1, \ldots, N$. For $i=-2,-1,(50)$ can be obtained by using (33)(41a), (41b), (41c), (41d), (41e), and (41f). By the definition $\mathscr{J}_{k}$, one obtains

$$
\mathscr{J}_{k}\left(f_{k \varepsilon}\right)-\mathscr{J}_{k}\left(f_{k}^{\circ}\right)=\sum_{j=1}^{P} d_{k j} \varepsilon_{j}+o(\varepsilon),
$$

where $\mathscr{J}_{k \varepsilon}$ is taken as $\mathscr{J}_{k}\left(f_{k \varepsilon}\right)$. This completes the proof of $D$ being a derived set for $Z$ at $\mathscr{J}_{k}\left(f_{k}^{\circ}\right)$. Then, there exist nonpositive Lagrange multipliers [36], not zero simultaneously, satisfying

$$
\sum_{i=-2}^{M} \lambda_{i k} d_{k}^{i} \leq 0, \quad \lambda_{0 k}=\lambda_{0}
$$

for any vector $d_{k}=\left(d_{k}^{-2}, d_{k}^{-1}, d_{k}^{0}, \ldots, d_{k}^{M}\right)$ in $D$. Let us take $P=1$ in the foregoing discussion to obtain the conclusion of Lemma 5 and put

$$
\overline{\mathscr{J}}_{k}=\sum_{i=-2}^{M} \lambda_{i k} \mathscr{J}_{i k}
$$

By (50), it follows that

$$
\overline{\mathscr{J}}_{k}\left(f_{k \varepsilon}\right)-\overline{\mathscr{J}}_{k}\left(f_{k}^{\circ}\right)=\varepsilon \sum_{i=-2}^{M} \lambda_{i k} d_{k}^{i}+o(\varepsilon),
$$

for any $d_{k}=\left(d_{k}^{-2}, d_{k}^{-1}, d_{k}^{0}, \ldots, d_{k}^{M}\right)$ in $D$. Then, we obtain the proof of Lemma 5 as follows:

$$
\lim _{\varepsilon \rightarrow 0^{+}} \frac{\overline{\mathscr{J}}_{k}\left(f_{k \varepsilon}\right)-\overline{\mathscr{J}}_{k}\left(f_{k}^{\circ}\right)}{\varepsilon}=\sum_{i=-2}^{M} \lambda_{i k} d_{k}^{i} \leq 0 .
$$

Theorem 6 (maximum principle). For the optimal control functions $f_{1}^{\circ}(x, t), \ldots, f_{N}^{\circ}(x, t) \in U_{a d}$, the corresponding optimal state and adjoint variables are $w^{\circ}(x, t)=$ $w\left(x, t, f_{1}^{\circ}(x, t), f_{2}^{\circ}(x, t), \ldots, f_{N}^{\circ}(x, t)\right)$ satisfying (1)-(4) and $v^{\circ}(x, t)=v\left(x, t, f_{1}^{\circ}(x, t), f_{2}^{\circ}(x, t), \ldots, f_{N}^{\circ}(x, t)\right)$ satisfying $(11)$, boundary conditions (13a) and (13b), and terminal conditions (14a) and (14b), respectively. The maximum principle states that if

$$
\begin{aligned}
\mathscr{H} & {\left[x, t, v^{\circ}, f_{1}^{\circ}(x, t), \ldots, f_{N}^{\circ}(x, t)\right] } \\
& =\max _{f_{k} \in U_{a d}} \mathscr{H}\left[x, t, v, f_{1}(x, t), \ldots, f_{N}(x, t)\right], \quad k=1, \ldots, N,
\end{aligned}
$$

where the Hamiltonian is given by

$$
\begin{aligned}
\mathscr{H}\left[x, t, v, f_{1}, \ldots, f_{N}\right]= & \sum_{k=1}^{N} v(x, t) f_{k} \\
& +\sum_{k=1}^{N} \sum_{i=-2}^{M} \lambda_{i k} \mathscr{G}_{i k}\left(x, t, w(x, t), f_{k}\right),
\end{aligned}
$$

then the performance index equation (8) is minimized; that is,

$$
\begin{array}{r}
\mathscr{J}_{0}\left[f_{1}^{\circ}(x, t), \ldots, f_{N}^{\circ}(x, t)\right] \leq \mathscr{J}_{0}\left[f_{1}(x, t), \ldots, f_{N}(x, t)\right] \\
\text { for any } f_{k} \in U_{a d} .
\end{array}
$$

Proof. Let $(x, t)$ be a regular point for admissible optimal control functions $f_{k}^{\circ}, k=1, \ldots, N$. By Lemma 5 , for $0 \leq$ $i \leq m$ and some $\lambda_{i k} \neq 0$, there exists Lagrange multipliers $\lambda_{-2 k}, \lambda_{-1 k}, \lambda_{0}, \ldots, \lambda_{N M}$ independent of $(x, t)$ with $\lambda_{i k} \leq 0$ such that

$$
\begin{gathered}
\sum_{k=1}^{N} \sum_{i=-2}^{M} \lambda_{i k}\left[v_{i k}(x, t)\left(f_{k}-f_{k}^{\circ}(x, t)\right)+\mathscr{G}_{i k}\left(x, t, w(x, t), f_{k}\right)\right. \\
\left.-\mathscr{G}_{i k}\left(x, t, w^{\circ}(x, t), f_{k}^{\circ}(x, t)\right)\right] \leq 0,
\end{gathered}
$$

for any function $f_{k} \in U_{\mathrm{ad}}$. Note that the term

$$
\sum_{k=1}^{N} \sum_{i=-2}^{M} \lambda_{i k} v_{i k}(x, t) f_{k}+\sum_{k=1}^{N} \sum_{i=-2}^{M} \lambda_{i k} \mathscr{G}_{i k}\left(x, t, w(x, t), f_{k}\right)
$$

reaches its maximum value at $f_{k}=f_{k}^{\circ}(x, t) \in U_{\text {ad }}$, for $k=$ $1, \ldots, N$. Let us consider the first term in (62),

$$
\sum_{k=1}^{N} \sum_{i=-2}^{M} \lambda_{i k} v_{i k}(x, t) f_{k}
$$

that can be rewritten in the form

$$
\sum_{k=1}^{N} \sum_{i=-2}^{M} \sum_{r=1}^{N} \lambda_{i k} v_{i k}(x, t) f_{r}
$$

to be subject to

$$
\lambda_{i k} v_{i k}(x, t) f_{r}= \begin{cases}\lambda_{i k} v_{i k}(x, t) f_{k}, & r=k, \\ 0, & r \neq k .\end{cases}
$$

If we define $v=\sum_{k=1}^{N} \sum_{i=-2}^{M} \lambda_{i k} v_{i k}(x, t)$, we obtain

$$
\sum_{k=1}^{N} v(x, t) f_{k}+\sum_{k=1}^{N} \sum_{i=-2}^{M} \lambda_{i k} \mathscr{G}_{i k}\left(x, t, w(x, t), f_{k}\right) \text {, }
$$

the conclusion of the maximum principle. This completes the proof of Theorem 6 . 
Theorem 7. Consider the control system equations (1)-(4) and (8)-(9a), (9b), (9c), and (9d). Let the functions $G_{i k}$ be in the form

$$
\begin{array}{r}
G_{i k}\left(x, t, w, f_{k}\right)=\mathscr{G}_{k}^{i}(x, t, w)+H_{k}^{i}\left(x, t, f_{k}\right), \\
i=-2,-1,0, \ldots, M, \quad k=1, \ldots, N
\end{array}
$$

and let $v$ satisfying (13a) and (13b)-(14a) and (14b) be the nonzero solution of

$$
\mathscr{M} v=\sum_{k=1}^{N} \sum_{i=-2}^{M} \lambda_{i k} \frac{\partial G_{k}^{i}\left(x, t, w^{\circ}(x, t)\right)}{\partial w}
$$

Assume that there exist admissible control functions $f_{1}^{\circ}, f_{2}^{\circ}, \ldots, f_{N}^{\circ}$ and the constants $\lambda_{0}, \lambda_{i k}, i=$ $-2,-1,1, \ldots, M, k=1, \ldots, N$ that satisfy the maximum principle equations (58). Let us assume that following assumptions are satisfied:

(a) for $k=1, \ldots, N, \mathscr{G}_{1}, h_{1 k}, \mathscr{G}_{i k}, \ldots, \mathscr{G}_{m k}$ are convex functions of $w$ and $\mathscr{G}_{2}, h_{2 k}$ are convex functions of $w_{t}$;

(b) $\lambda_{0}<0, \quad \lambda_{i k} \leq 0$, for $i=-1, \ldots, m, k=1, \ldots, N$;

(c) the constraints equation (9a), (9b), (9c), and (9d) are satisfied by $f_{1}^{\circ}, f_{2}^{\circ}, \ldots, f_{N}^{\circ}$

(d) if the strict inequality holds in (9a), (9b), (9c), and (9d), the corresponding Lagrange multiplier $\lambda_{i k}=0$;

(e) $-\lambda_{i k} G_{k}^{i},-\lambda_{-1 k} h_{1 k}$ are convex functions of $w$ and $-\lambda_{-2 k} h_{2 k}$ is convex function of $w_{t}$, for $k=1, \ldots$, $N, m<i<M$.

Under these assumptions, the maximum principle given by (58) is also sufficient condition for the admissible control functions $f_{1}^{\circ}, f_{2}^{\circ}, \ldots, f_{N}^{\circ}$ to be optimal. The condition $(d)$ is proved in [36]. If the $h_{1 k}, G_{i k}$, and $h_{2 k}$ are linear functions of $w$ and $w_{t}$, respectively, the condition (e) is satisfied, for $k=1, \ldots, N, m<$ $i \leq M$.

Proof. If $f_{k}$ 's and $w$ satisfy (9a), (9b), (9c), and (9d), then, by the condition $(\mathrm{d})$,

$$
\begin{aligned}
& \int_{0}^{t_{f}} \int_{0}^{\ell} \lambda_{i k}\left[G_{k}^{i}(x, t, w)-G_{k}^{i}\left(x, t, w^{\circ}\right)\right] d x d t \\
& \quad+\int_{0}^{t_{f}} \int_{0}^{\ell} \lambda_{i k}\left[H_{k}^{i}\left(x, t, f_{k}\right)-H_{k}^{i}\left(x, t, f_{k}^{\circ}\right)\right] d x d t=0,
\end{aligned}
$$

for $i=-2,-1, \ldots, M, k=1, \ldots, N$. Then, we can write the following inequality

$$
\begin{aligned}
&- \lambda_{0}\left[\mathscr{J}_{0}\left(f_{1}, f_{2}, \ldots, f_{N}\right)-\mathscr{J}_{0}\left(f_{1}^{\circ}, f_{2}^{\circ}, \ldots, f_{N}^{\circ}\right)\right] \\
& \geq-\int_{0}^{\ell} \lambda_{0}\left[\mathscr{G}_{2}\left(x, w_{t}\left(x, t_{f}\right)\right)-\mathscr{G}_{2}\left(x, w_{t}^{\circ}\left(x, t_{f}\right)\right)\right. \\
&\left.\quad+\mathscr{G}_{1}\left(x, w\left(x, t_{f}\right)\right)-\mathscr{G}_{1}\left(x, w^{\circ}\left(x, t_{f}\right)\right)\right] d x .
\end{aligned}
$$

$$
\begin{gathered}
-\int_{0}^{t_{f}} \int_{0}^{\ell} \sum_{k=1}^{N} \sum_{i=-2}^{M} \lambda_{i k}\left\{G_{k}^{i}(x, t, w(x, t))-G_{k}^{i}\left(x, t, w^{\circ}(x, t)\right)\right. \\
-\left[H_{k}^{i}\left(x, t, f_{k}(x, t)\right)\right. \\
\left.\left.-H_{k}^{i}\left(x, t, f_{k}^{\circ}(x, t)\right)\right]\right\} d x d t \\
-\int_{0}^{\ell} \sum_{k=1}^{N} \lambda_{-1 k}\left[h_{1 k}\left(x, w\left(x, t_{f}\right)\right)-h_{1 k}\left(x, w^{\circ}\left(x, t_{f}\right)\right)\right] d x \\
-\int_{0}^{\ell} \sum_{k=1}^{N} \lambda_{-2 k}\left[h_{2 k}\left(x, w_{t}\left(x, t_{f}\right)\right)-h_{2 k}\left(x, w_{t}^{\circ}\left(x, t_{f}\right)\right)\right] d x
\end{gathered}
$$

By using the convexity assumption (e),

$$
\begin{aligned}
& -\lambda_{0}\left[\mathscr{J}_{0}\left(f_{1}, f_{2}, \ldots, f_{N}\right)-\mathscr{J}_{0}\left(f_{1}^{\circ}, f_{2}^{\circ}, \ldots, f_{N}^{\circ}\right)\right] \\
& \geq-\int_{0}^{\ell} \lambda_{0}\left[\frac{\partial \mathscr{G}_{1}}{\partial w}\left(x, w^{\circ}\left(x, t_{f}\right) \Delta w\left(x, t_{f}\right)\right)\right. \\
& \left.+\frac{\partial \mathscr{G}_{2}}{\partial w_{t}}\left(x, w_{t}^{\circ}\left(x, t_{f}\right) \Delta w_{t}\left(x, t_{f}\right)\right)\right] d x \\
& -\int_{0}^{t_{f}} \int_{0}^{\ell} \sum_{k=1}^{N} \sum_{i=-2}^{M} \lambda_{i k} \frac{\partial G_{k}^{i}}{\partial w}\left(x, t, w^{\circ}(x, t)\right) \Delta w(x, t) d x d t \\
& +\int_{0}^{t_{f}} \int_{0}^{\ell} \sum_{k=1}^{N} \sum_{i=-2}^{M} \lambda_{i k}\left[H_{k}^{i}\left(x, t, f_{k}^{\circ}(x, t)\right)\right. \\
& \left.-H_{k}^{i}\left(x, t, f_{k}(x, t)\right)\right] d x d t \\
& -\int_{0}^{\ell} \sum_{k=1}^{N} \lambda_{-1 k} \frac{\partial h_{1 k}}{\partial w}\left(x, w^{\circ}\left(x, t_{f}\right)\right) \Delta w\left(x, t_{f}\right) d x \\
& -\int_{0}^{\ell} \sum_{k=1}^{N} \lambda_{-2 k} \frac{\partial h_{2 k}}{\partial w_{t}}\left(x, w_{t}^{\circ}\left(x, t_{f}\right)\right) \Delta w_{t}\left(x, t_{f}\right) d x \\
& =-\int_{0}^{\ell} \lambda_{0}\left[\frac{\partial \mathscr{G}_{1}}{\partial w}\left(x, w^{\circ}\left(x, t_{f}\right)\right) \Delta w\left(x, t_{f}\right)\right. \\
& \left.+\frac{\partial \mathscr{G}_{2}}{\partial w_{t}}\left(x, w_{t}^{\circ}\left(x, t_{f}\right)\right) \Delta w_{t}\left(x, t_{f}\right)\right] d x \\
& -\int_{0}^{t_{f}} \int_{0}^{\ell}(\mathscr{M} v) \Delta w(x, t) d x d t \\
& +\int_{0}^{t_{f}} \int_{0}^{\ell} \sum_{k=1}^{N} \sum_{i=-2}^{M} \lambda_{i k}\left[H_{k}^{i}\left(x, t, f_{k}^{\circ}(x, t)\right)\right. \\
& \left.-H_{k}^{i}\left(x, t, f_{k}(x, t)\right)\right] d x d t \\
& -\int_{0}^{\ell} \sum_{k=1}^{N}\left[\lambda_{-1 k} \frac{\partial h_{1 k}}{\partial w}\left(x, w^{\circ}\left(x, t_{f}\right)\right) \Delta w\left(x, t_{f}\right)\right. \\
& \left.+\lambda_{-2 k} \frac{\partial h_{2 k}}{\partial w_{t}}\left(x, w_{t}^{\circ}\left(x, t_{f}\right)\right) \Delta w_{t}\left(x, t_{f}\right)\right] d x .
\end{aligned}
$$


And finally by applying Lemma 2 and the conditions (13a) and (13b)-(14a) and (14b), we obtain

$$
\begin{gathered}
-\lambda_{0}\left[\mathscr{F}_{0}\left(f_{1}, f_{2}, \ldots, f_{N}\right)-\mathscr{F}_{0}\left(f_{1}^{\circ}, f_{2}^{\circ}, \ldots, f_{N}^{\circ}\right)\right] \\
\geq \int_{0}^{t_{f}} \int_{0}^{\ell}\left\{\sum_{k=1}^{N} v(x, t)\left[f_{k}^{\circ}(x, t)-f_{k}(x, t)\right]\right. \\
+\sum_{k=1}^{N} \sum_{i=-2}^{M} \lambda_{i k}\left[H_{k}^{i}\left(x, t, f_{k}^{\circ}(x, t)\right)\right. \\
\left.\left.-H_{k}^{i}\left(x, t, f_{k}(x, t)\right)\right]\right\} d x d t .
\end{gathered}
$$

Note that the right-hand side of (72) is nonnegative due to the condition (b). Then, we obtain

$$
\begin{aligned}
\mathscr{J}_{0}( & \left.f_{1}(x, t), f_{2}(x, t), \ldots, f_{N}(x, t)\right) \\
& \quad-\mathscr{J}_{0}\left(f_{1}^{\circ}(x, t), f_{2}^{\circ}(x, t), \ldots, f_{N}^{\circ}(x, t)\right) \geq 0 .
\end{aligned}
$$

It follows that the maximum principle is also a sufficient condition for a global minimum of the performance index (8). It completes the proof of Theorem 7.

\section{Conclusion}

A necessary optimality condition for a general class of damped hyperbolic partial differential equation in one-space dimension is derived in the form of a maximum principle by using the derived set and regular point concepts. Under proper convexity assumption on state variable, it is proved thatthe maximum principle is also sufficient condition for the controls to be optimal . In [1-7], the systems under consideration include only one control function. Also, the necessary and sufficient conditions for optimality are given for a single hyperbolic differential equation without damping term subject to the homogeneous boundary conditions in onespace dimension. But, in the present paper, as an original contribution to literature, the necessary and sufficient optimality conditions obtained in [1-7] are generalized for a general class of damped hyperbolic equation involving damping and several control functions subject to nonhomogeneous boundary conditions in one space dimension.

\section{Conflict of Interests}

The authors declare that there is no conflict of interests regarding the publication of this paper.

\section{References}

[1] L. S. Pontryagin, V. Boltyanskii, R. Gamkrelidze, and E. Mishchenko, The Mathematical Theory of Optimal Control Processes, Edited by: L. W. Neustadt, Interscience, New York, NY, USA, 1962.

[2] E. B. Lee, "A sufficient condition in the theory of optimal control," SIAM Journal on Control, vol. 1, pp. 241-245, 1963.
[3] A. I. Egorov, "Necessary optimality conditions for distributedparameter systems," SIAM Journal on Control and Optimization, vol. 5, pp. 352-408, 1967.

[4] F. H. Clarke, "The maximum principle under minimal hypotheses," SIAM Journal on Control and Optimization, vol. 14, no. 6, pp. 1078-1091, 1976.

[5] F. Clarke, "Necessary conditions in dynamic optimization," Memoirs of the American Mathematical Society, vol. 173, no. 816, 2005.

[6] B. Sun, "Maximum principle for optimal boundary control of the Kuramoto-Sivashinsky equation," Journal of the Franklin Institute. Engineering and Applied Mathematics, vol. 347, no. 2, pp. 467-482, 2010.

[7] E. R. Barnes, "Necessary and sufficient optimality conditions for a class of distributed parameter control systems," SIAM Journal on Control and Optimization, vol. 9, pp. 62-82, 1971.

[8] A. V. Dmitruk, "Maximum principle for the general optimal control problem with phase and regular mixed constraints," Computational Mathematics and Modeling, vol. 4, no. 4, pp. 364-377, 1993.

[9] A. Ya. Dubovitskiĭ and A. A. Milyutin, "Theory of the principle of the maximum," in Methods of the Theory of Extremal Problems in Economics, pp. 7-47, Nauka, Moscow, Russia, 1981.

[10] H. J. Sussmann, "Strong version of the Maximum Principle under weak hypotheses," in Proceedings of the 33rd IEEE Conference on Decision and Control, pp. 1950-1956, December 1994.

[11] J. Warga, "Necessary conditions without differentiability assumptions in optimal control," Journal of Differential Equations, vol. 18, pp. 41-62, 1975.

[12] F. H. Clarke, "Maximum principles without differentiability," Bulletin of the American Mathematical Society, vol. 81, pp. 219222, 1975 .

[13] S. Ilter, "Weak maximum principle for optimal control problems of nonsmooth systems," Applied Mathematics and Computation, vol. 218, no. 3, pp. 805-808, 2011.

[14] A. A. Agrachev and R. V. Gamkrelidze, "The geometry of the maximum principle," Proceedings of the Steklov Institute of Mathematics, vol. 273, no. 1, pp. 1-22, 2011.

[15] M. D. R. de Pinho and A. Ilchmann, "Weak maximum principle for optimal control problems with mixed constraints," Nonlinear Analysis: Theory, Methods \& Applications, vol. 48, no. 8, pp. 1179-1196, 2002.

[16] S. O. Lopes, F. A. C. C. Fontes, and M. d. R. de Pinho, "An integral-type constraint qualification to guarantee nondegeneracy of the maximum principle for optimal control problems with state constraints," Systems \& Control Letters, vol. 62, no. 8, pp. 686-692, 2013

[17] A. V. Arutyunov, D. Yu. Karamzin, and F. Pereira, "Pontryagin's maximum principle for constrained impulsive control problems," Nonlinear Analysis: Theory, Methods \& Applications, vol. 75, no. 3, pp. 1045-1057, 2012.

[18] P. Felmer, M. Montenegro, and A. Quaas, "A note on the strong maximum principle and the compact support principle," Journal of Differential Equations, vol. 246, no. 1, pp. 39-49, 2009.

[19] M. E. Mincsovics, "Discrete and continuous maximum principles for parabolic and elliptic operators," Journal of Computational and Applied Mathematics, vol. 235, no. 2, pp. 470-477, 2010.

[20] I. A. Shvartsman, "New approximation method in the proof of the maximum principle for nonsmooth optimal control problems with state constraints," Journal of Mathematical Analysis and Applications, vol. 326, no. 2, pp. 974-1000, 2007. 
[21] M. McAsey and L. Mou, "A proof of a general maximum principle for optimal controls via a multiplier rule on metric space," Journal of Mathematical Analysis and Applications, vol. 337, no. 2, pp. 1072-1088, 2008.

[22] F. H. Wong, C. C. Yeh, and S. L. Yu, "A maximum principle forsecond order nonlinear differential inequalities and its applications," Applied Mathematics Letters, vol. 8, no. 4, pp. 91-96, 1995.

[23] D. E. Chang, "A simple proof of the Pontryagin maximum principle on manifolds," Automatica, vol. 47, no. 3, pp. 630-633, 2011.

[24] J. Shen, J. Li, and C. Zou, "Note on maximum principles of first order impulsive boundary value problems," Applied Mathematics Letters, vol. 19, no. 11, pp. 1159-1161, 2006.

[25] P. Stehlik and B. Thompson, "Maximum principles for second order dynamic equations on time scales," Journal of Mathematical Analysis and Applications, vol. 331, no. 2, pp. 913-926, 2007.

[26] H. Hermes, "Asymptotically stabilizing feedback controls," Journal of Differential Equations, vol. 92, no. 1, pp. 76-89, 1991.

[27] E. Moulay, "Stabilization via homogeneous feedback controls," Automatica, vol. 44, no. 11, pp. 2981-2984, 2008.

[28] A. R. Teel, "Global stabilization and restricted tracking for multiple integrators with bounded controls," Systems \& Control Letters, vol. 18, no. 3, pp. 165-171, 1992.

[29] R. O. Saber and R. M. Murray, "Distributed cooperative control of multiple vehicle formations using structural potential function," in Proceedings of the IFAC World Congress, 2002.

[30] W. Ren and R. W. Beard, Distributed Consensus in Multi-Vehicle Cooperative Control, Communications and Control Engineering Series, Springer, London, UK, 2008.

[31] J. M. Sloss, I. S. Sadek, J. C. Bruch Jr., and S. Adali, "Maximum principle for the optimal control of a hyperbolic equation in one space dimension. I. Theory," Journal of Optimization Theory and Applications, vol. 87, no. 1, pp. 33-45, 1995.

[32] E. C. Zachmanoglou and D. W. Thoe, Introduction to Partial Differential Equations with Applications, Dover, New York, NY, USA, 2nd edition, 1986.

[33] N. S. Koshlyakov, M. M. Smirnov, and E. B. Gliner, Differential Equations of Mathematical Physics, North-Holland, Amsterdam, The Netherlands, 1964.

[34] I. S. Sadek, "Necessary and sufficient conditions for the optimal control of distributed parameter systems subject to integral constraints," Journal of the Franklin Institute, vol. 325, no. 5, pp. 565-583, 1988.

[35] S. Saks, Theory of the Integral, Hafner, New York, NY, USA, 1937.

[36] M. R. Hestenes, Calculus of Variations and Optimal Control Theory, John Wiley \& Sons, New York, NY, USA, 1966. 


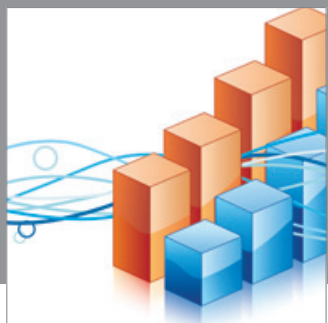

Advances in

Operations Research

mansans

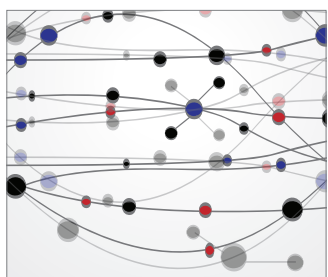

The Scientific World Journal
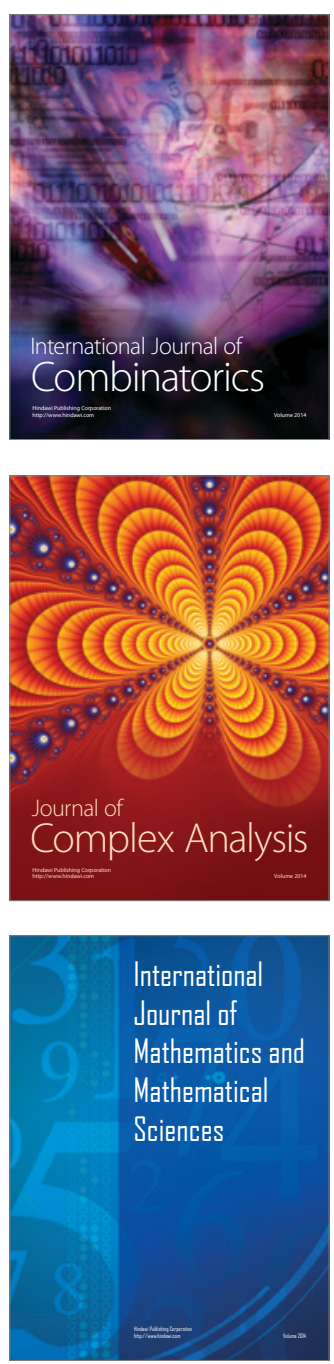
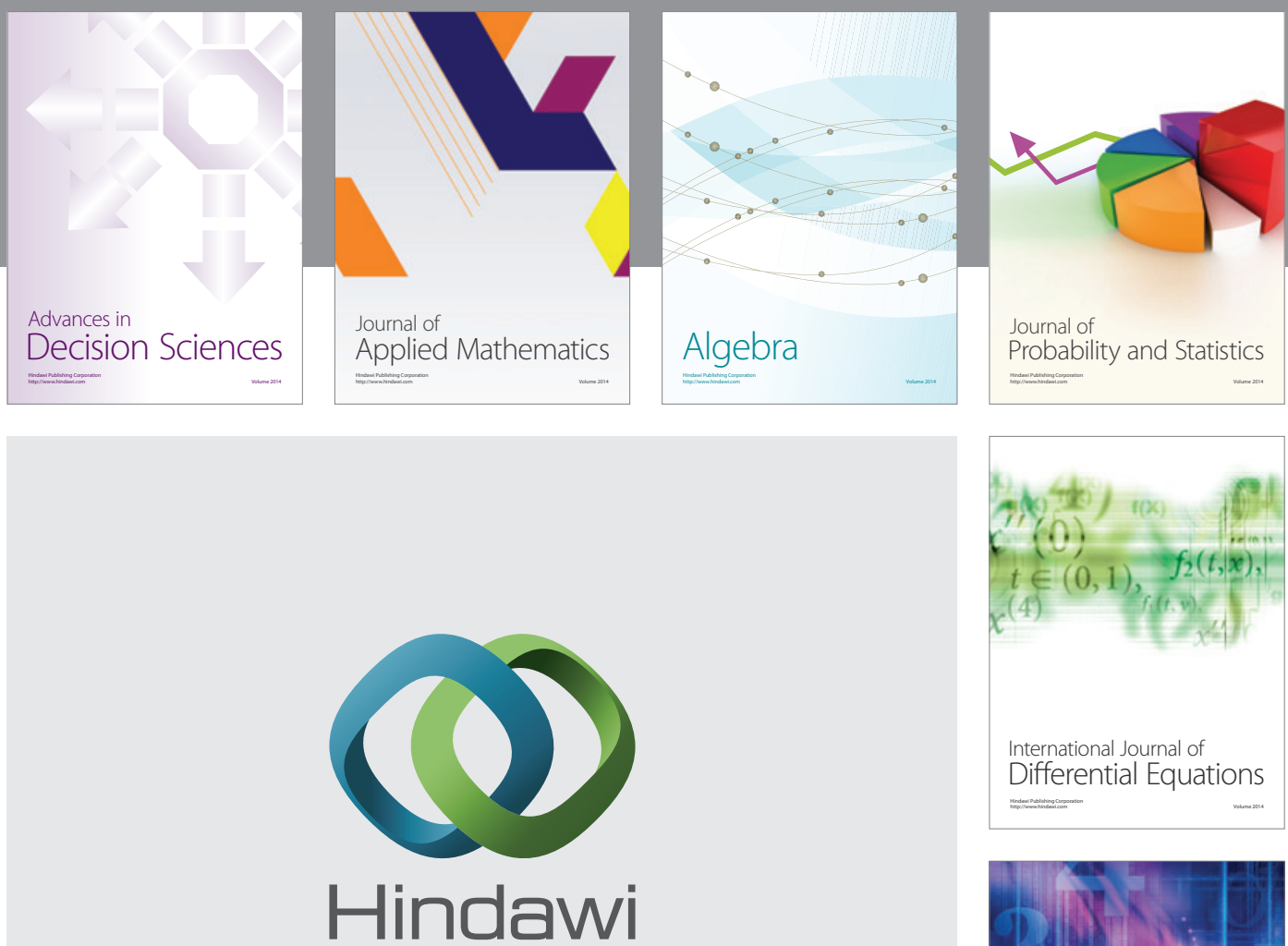

Submit your manuscripts at http://www.hindawi.com
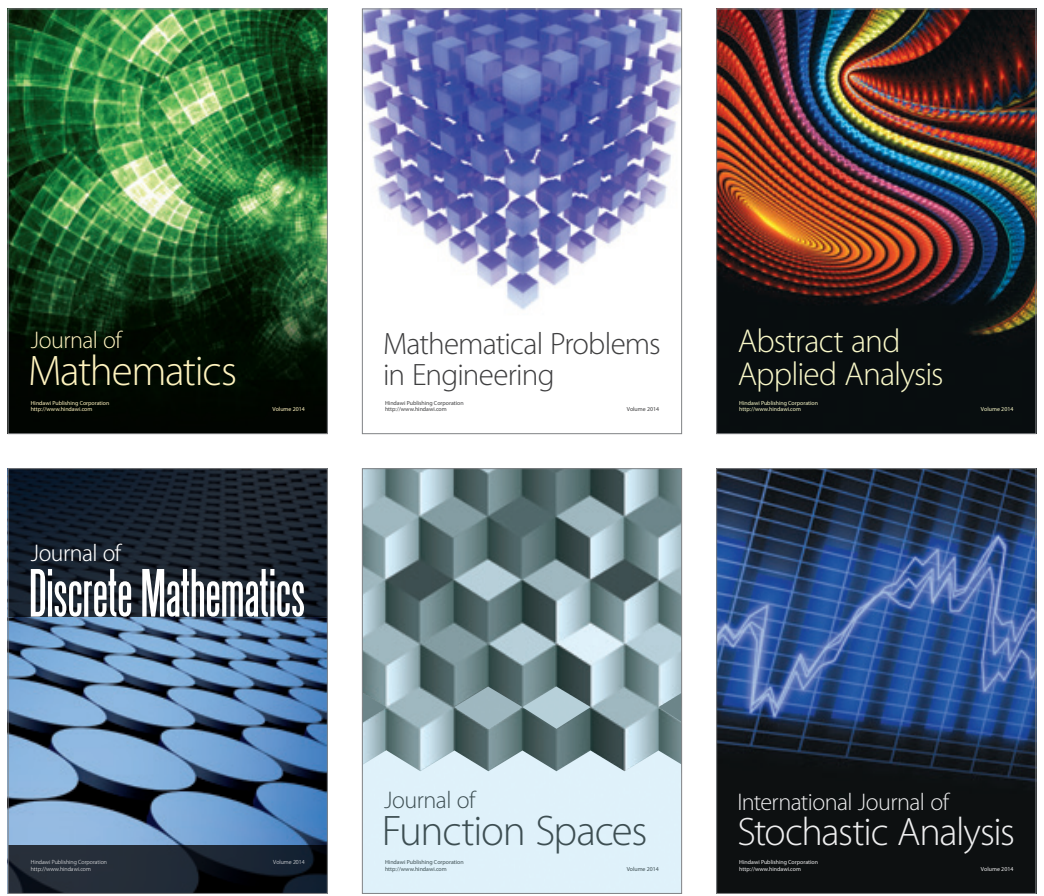

Journal of

Function Spaces

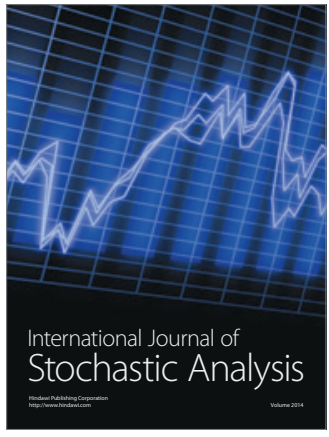

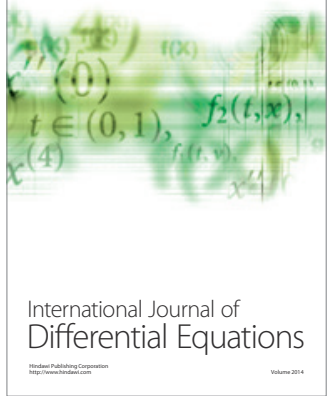
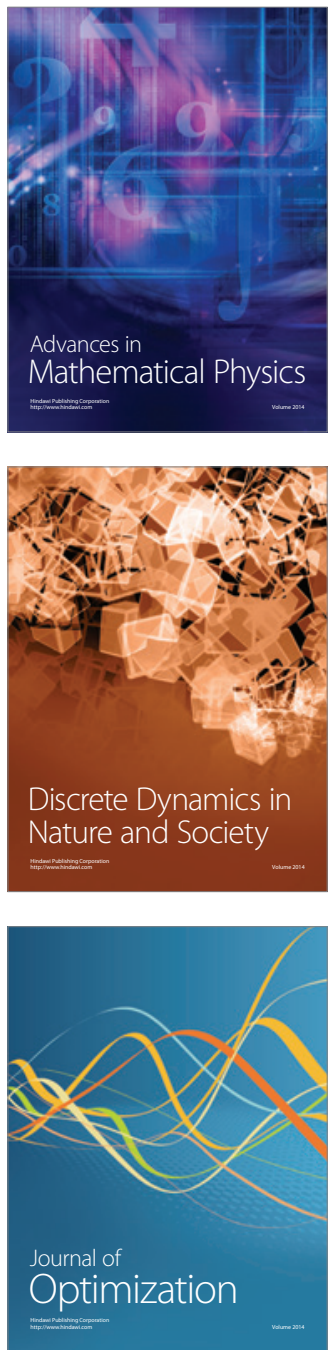